\title{
ChemComm
}

COMMUNICATION

\section{Dynamic flow synthesis of porous organic cages}

Received 00th January 20xx, Accepted 00th January 20xx

DOI: $10.1039 / x 0 x \times 00000 x$

\author{
Michael E. Briggs ${ }^{\dagger a}$, Anna G. Slater ${ }^{\dagger a}$, Neil Lunt ${ }^{a}$, Shan Jiang ${ }^{a}$, Marc A. Little ${ }^{a}$, Rebecca L.
} Greenaway $^{a}$, Tom Hasell ${ }^{a}$, Claudio Battilocchio ${ }^{b}$, Steven V. Ley ${ }^{b}$ and Andrew I. Cooper*a

www.rsc.org/

The dynamic covalent synthesis of two imine-based porous organic cages was successfully transferred from batch to continuous flow. The same flow reactor was then used to scramble the constituents of these two cages in differing ratios to form cage mixtures. Preparative HPLC purification of one of these mixtures allowed rapid access to a desymmetrised cage molecule.

Porous organic cages (POCs) are discrete, covalently bonded molecules that possess intrinsic, shape-persistent voids, or 'molecular pores'..$^{1-3}$ Porosity in the bulk material results when these intrinsic voids are interconnected to form a guestaccessible pore network. POCs can be isolated as either amorphous or crystalline solids, and the molecular packing in the material has a major impact on its properties because it defines how the intrinsic cage voids are connected. ${ }^{4}$ Changes to the outer periphery of the cage can alter both the crystal packing and the topology of the pore network. ${ }^{5,6}$ For instance, CC1 $\alpha$ was found to be formally non-porous because the intrinsic cage cavities are isolated in the crystal structure; by contrast, the cavities in $\mathbf{C C} 3 \alpha$ are interconnected by a 3dimensional (3-D) pore network that results in an apparent Brunauer-Emmett-Teller surface area $\left(\mathrm{SA}_{\mathrm{BET}}\right)$ of $410 \mathrm{~m}^{2} / \mathrm{g}$ for crystalline CC3 $\alpha$ (Scheme 1, Figure S1). ${ }^{4}$ To date POCs have found applications in molecular separations, ${ }^{7-9}$ sensing, ${ }^{10,11}$ and as catalyst supports, ${ }^{12}$ and surface areas of up to $3758 \mathrm{~m}^{2} / \mathrm{g}$ have been achieved. ${ }^{13}$ However, there are still few examples of POCs in the literature compared to other established classes of porous materials such as zeolites, metal-organic frameworks (MOFs), and porous organic polymers. ${ }^{14}$

Historically, macrocyclic molecules such as organic cages have often been prepared under high dilution conditions, ${ }^{15-17}$ which are inconvenient for larger scales. The development of

\footnotetext{
${ }^{a}$ Department of Chemistry and Centre for Materials Discovery, University of Liverpool, Crown Street, Liverpool, L69 7ZD, UK. E-mail:aicooper@liverpool.ac.uk; Web: http://www.liv.ac.uk/cooper-group/

${ }^{b}$ Innovative Technology Centre, Department of Chemistry, University of Cambridge, Lensfield Road, Cambridge, CB2 1EW, UK.

TMEB and AGS contributed equally to the preparation of this manuscript. Electronic Supplementary Information (ESI) available: Full synthetic and crystallographic details. CCDC 1411292. See DOI: 10.1039/x0xx00000x
}

continuous flow processes is therefore an attractive opportunity, both for combinatorial discovery of new cages and for subsequent scale-up. Unlike porous frameworks such as zeolites and MOFs, POCs are soluble in many common organic solvents; this property allows their isolation to be decoupled from their synthesis, making them ideal candidates for flow chemistry. ${ }^{18,19}$

Continuous flow systems have become increasingly popular as an alternative to conventional batch reactions. ${ }^{20-23}$ The increased availability of commercial flow reactors has allowed flow chemistry to evolve from a niche technique into a mainstream tool. This has been driven by the promise of precise control of reaction parameters and faster kinetics, combined with the ability to rapidly screen and optimise reaction conditions. Here, the ability to quickly evaluate the potential of new precursors to form cages, while at the same time offering a facile method for scale-up, would be of tremendous benefit and could accelerate the rapid discovery and characterisation of new functional POCs.

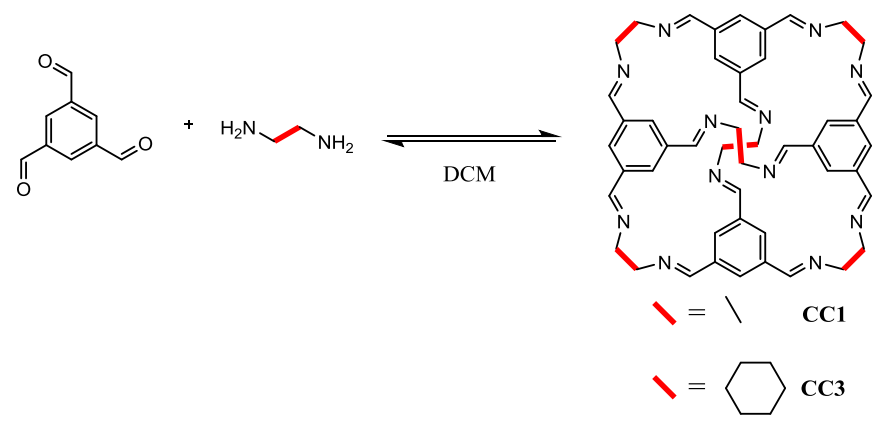

Scheme 1 Synthesis of CC1 and CC3- $R$ (The italicised letter denotes the chirality of the 1,2-cyclohexanediamine starting material, in this case $R, R$. The opposite cage enantiomer, CC3-S, is formed from S,S-1,2-cyclohexanediamine.)

As a proof-of-concept, we transferred the synthesis of two known imine-based POCs, CC1 and CC3-R (Scheme 1), onto a Vapourtec R-series flow reactor system. Each cage is synthesised through the formation of 12 imine bonds via the reaction of 4 trialdehydes and 6 diamines in a dynamic 
covalent cycloimination reaction; the reversibility of the reaction provides an error-correction mechanism that allows conversion of any kinetic products to the thermodynamic cage products. $^{24}$ Without this error correction mechanism, oligomeric or polymeric side products could form and precipitate irreversibly from the reaction mixture. For dynamic covalent synthesis to succeed the target compound should be the thermodynamic product. Also, all intermediates should remain in solution and the reaction should exhibit sufficient reversibility on the timescale of the reaction for the thermodynamic product to be obtained. To meet these criteria in batch syntheses, organic cages and macrocycles are often synthesised at high dilution with slow reactant addition in the presence of a suitable catalyst. ${ }^{17,25}$

With the exception of simple bimolecular imine forming reactions, ${ }^{26}$ there are, to the best of our knowledge, no examples in the literature of dynamic covalent chemistry occurring in flow. Very recently, Doonan et al. reported the first synthesis of a POC in flow via irreversible alkyne homocoupling; ${ }^{27}$ we believe this to be the only other example of a POC synthesised in flow.

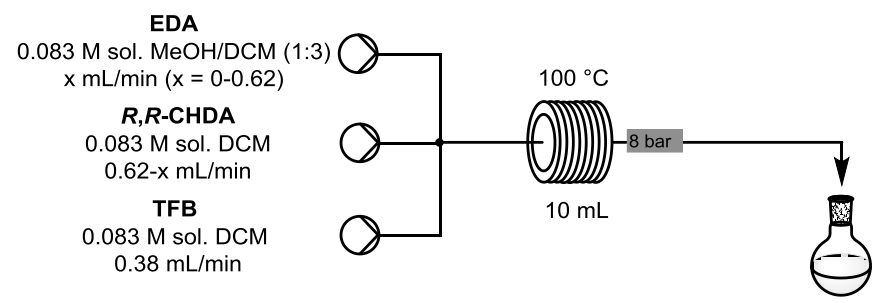

Fig. 1 Schematic representation of the synthesis of CC1 $(x=0.62)$, CC3-R $(x=0)$ and scrambled cages $(x=0-0.62)$ using the flow reactor.

Our initial work focused on optimising the continuous flow synthesis of CC3- $R$ and developing an understanding of the effect of temperature, flow rate, and residence time on the reaction. Solutions of 1,3,5-triformylbenzene (TFB, $0.083 \mathrm{M}$ ) and $R, R-1,2$-cyclohexanediamine (CHDA, $0.083 \mathrm{M}$ ) in dichloromethane (DCM) were prepared. The limited solubility of TFB prevented the use of more concentrated solutions. The reactant stoichiometry was fixed at 4:6 TFB to CHDA by controlling the relative flow rates, with the two reactant streams being combined in a T-piece before entering the reactor. Each reaction was sampled once the steady state had been reached (i.e., after $\sim 1.5 x$ the reactor volume of solvent had eluted) and analysed off-line using HPLC. Over the temperature range $40-120^{\circ} \mathrm{C}$, all intermediates and the CC3- $R$ product remained in solution (Table S1, fig. S3). However, at higher temperatures $\left(>120^{\circ} \mathrm{C}\right)$, insoluble materials were produced. At $100{ }^{\circ} \mathrm{C}$, a residence time of $6 \mathrm{~min}$ was sufficient for complete conversion of the reactants to CC3- $R$; shorter residence times or lower temperatures afforded incomplete reactions, whereas longer residence times had no effect on the outcome (Table S1, fig. S4). Varying the flow rate over the range $0.2-2.0 \mathrm{~mL} / \mathrm{min}$, while maintaining the temperature and residence time at $100{ }^{\circ} \mathrm{C}$ and $10 \mathrm{~min}$, respectively, also had no effect on the reaction outcome (Table S1, fig. S5). Initially, minor changes in the reactant stoichiometry-possibly due to fluctuations in pump performance or precipitation of CHDA carbamate salts through reaction with atmospheric $\mathrm{CO}_{2}$-were found to result in incomplete conversions. Upon further investigation, we found that the cage forming reaction was sensitive to the presence of excess aldehyde, while excess CHDA was well tolerated, and afforded complete conversion of the aldehyde to cage (Table S1, fig. S6). Hence, to compensate for any minor changes in reactant stoichiometry, a ratio of 4:6.5 TFB to diamine was adopted for all subsequent reactions. The use of excess diamine resulted in an increase in the reproducibility and robustness of the reaction. The cage product was then precipitated directly from the reaction mixture by adding hexane to the reaction stream once it had passed through the back-pressure regulator (BPR). We were surprised to find that the optimised conditions for CC3- $R$ $(0.62 \mathrm{~mL} / \mathrm{min}$ CHDA $(0.083 \mathrm{M}$ in DCM); $0.38 \mathrm{~mL} / \mathrm{min}$ TFB $(0.083 \mathrm{M}$ in $\mathrm{DCM}) ; 10 \mathrm{~min}$ residence time; $100^{\circ} \mathrm{C}$ ) also worked well for the synthesis of $\mathbf{C C 1}$, despite the significant differences between the batch synthesis conditions for these two cages, and the reduced thermal and hydrolytic stability of CC1. Our only modification to the CC3- $R$ procedure was that the ethylenediamine (EDA) was dissolved in a 1:3 methanol/DCM mixture, rather than in neat DCM, because this was found to give more reliable pump performance. Again, the cage, CC1, was precipitated by addition of the concentrated reaction mixture into hexane.

For both cages, HPLC analysis of the reaction streams on exiting the reactor showed a purity of $>99 \%$ by peak area, with this high purity being maintained on isolation. Comparison of the batch and flow synthesis for CC1 and CC3- $R$ (Figure 2) showed a significant reduction in the reaction times while maintaining high isolated yields and product purity. In particular, the successful transfer of the CC1 synthesis to flow conditions highlights the potential of using flow reactors instead of high dilution, low temperature batch reactions. Using a single $10 \mathrm{~mL}$ reactor, it proved possible to isolate $0.35 \mathrm{~g} / \mathrm{h}$ of CC1 in a yield of $93 \%$, and $0.50 \mathrm{~g} / \mathrm{h}$ of CC3 in a yield of $95 \%$; batch reaction times for the two cages are 3 and 5 days respectively with yields of $94 \%$ (CC1) and $83 \%$ (CC3). The significant reduction in the reaction times is due to the ability to heat DCM at $100^{\circ} \mathrm{C}$ (using back-pressure regulators) alongside improved mixing and heat transfer.

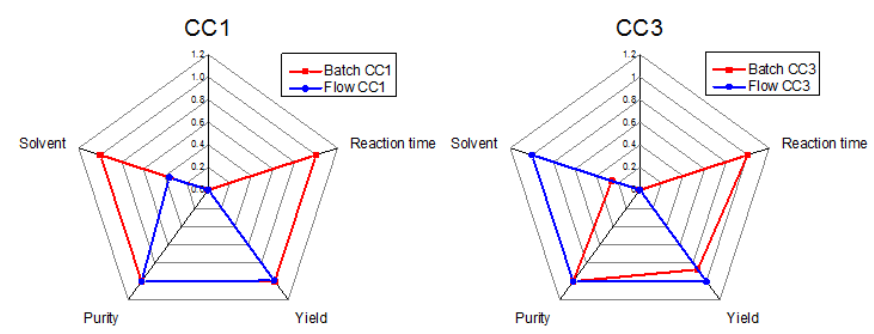

Fig. 2 Comparison of the reaction conditions employed for the synthesis of CC3 $R$ and CC1 in batch (blue) and flow (red), normalised for clarity.

Once the flow synthesis of CC1 and CC3- $R$ had been optimised, we turned our attention to the synthesis of scrambled mixtures of the two cages. We previously reported 
that the EDA and CHDA vertices in CC1 and CC3- $R$ can be interchanged without affecting the underlying tetrahedral cage structure. ${ }^{28}$ The closely matched geometries of EDA and CHDA favour dynamic covalent scrambling over the narcissistic or social self-sorting reported by the groups of Beurerle ${ }^{29}$ and Mukherjee. ${ }^{30}$ As such, it is possible to prepare scrambled cage mixtures containing a wide range of ratios of EDA and CHDA (Fig. S17). Small changes in the ratio of the diamines can have a profound effect on the physical properties of the cage mixtures; for example, we previously reported that porosity and gas selectivity could be tuned by varying the proportion of EDA and CHDA in the scrambled cages. ${ }^{28}$

Here, it proved possible to access scrambled cage mixtures with different properties by using the flow system to control the EDA to CHDA ratio. While maintaining the same 4:6.5 TFB to diamine ratio, we dosed in all of the possible whole number ratios of EDA:CHDA from 6:0 through to 0:6. A thermodynamic distribution of cages was produced, with the most abundant cage closely matching the diamine stoichiometry in the reactant feed (Fig. 3). The product distribution can be instantly shifted by changing the relative pumping rates, and hence the ratio of the diamine reactants. This is broadly consistent with what was previously reported when the reaction was run in batch, although differences in reactant stoichiometry and sampling mean the distributions are not identical.

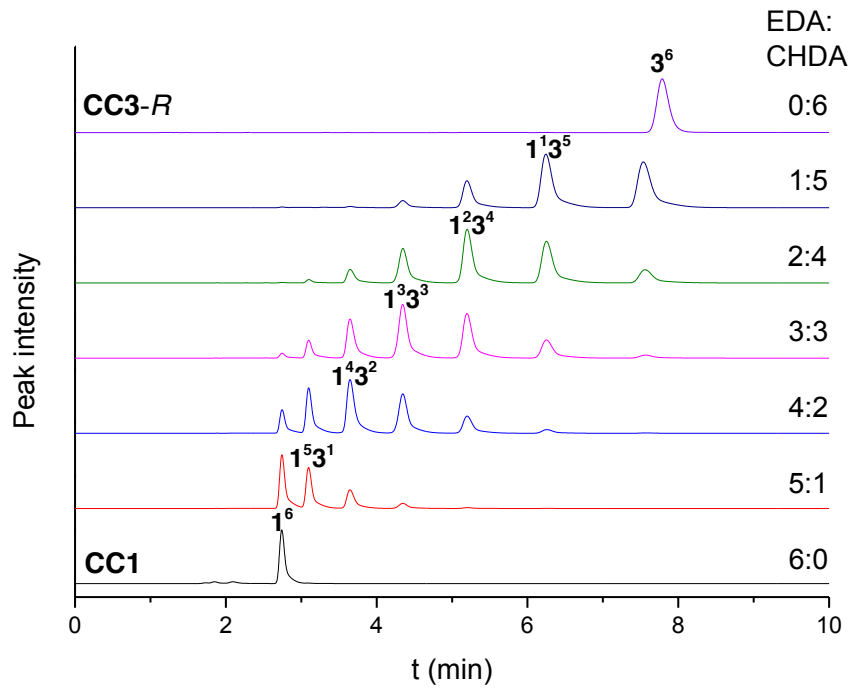

Fig. 3 Off-line HPLC analysis of cages CC1, CC3, and mixtures containing all ratios of EDA to CHDA The product distribution can be shifted by changing the diamine of EDA to CHDA. The product
ratio using the flow system.

Dynamic covalent scrambling was employed as a strategy to isolate individual desymmetrised cages, which are much harder to access than symmetrical cages. For example, the cage comprising $1 \mathrm{EDA}$ and $5 \mathrm{CHDA}, \mathrm{CC1}^{1} 3^{5}$, possesses a single ethylene bridged diimine that is an attractive target for selective monofunctionalisation (Fig. 4). Running the flow reactor with a 1:5 EDA to CHDA diamine ratio (Fig. 2, $x=0.103$ ) afforded the scrambled cage mixture with the greatest proportion of $\mathrm{CC1}^{1} 3^{5}$ (Fig. 3; second HPLC trace from the top). The resulting scrambled cage mixture was purified using reverse phase preparative HPLC to afford $\mathrm{CC1}^{1} 3^{5}$ in a yield of
$21 \%$ with a purity of $97 \%$ by peak area (Fig. S27). In solution $\mathrm{CC1}^{1} 3^{5}$ can slowly re-equilibrate back to the pre-purification cage distribution (Fig. 3, second HPLC trace from the top)-this can be accelerated by heating or the presence of acid. The discarded scrambled cage fractions could, in principle, also be re-equilibrated to the pre-purification cage distribution, which could then be re-purified by preparative HPLC in order to increase the overall yield of the $\operatorname{CC1}^{1} 3^{5}$. However, while reequilibration is observed in solution, once isolated in the solid state no re-equilibration of $\mathbf{C C 1}^{1} 3^{5}$ was observed. The structure of the desymmetrised cage was confirmed by single crystal X-ray diffraction (Fig. 4).

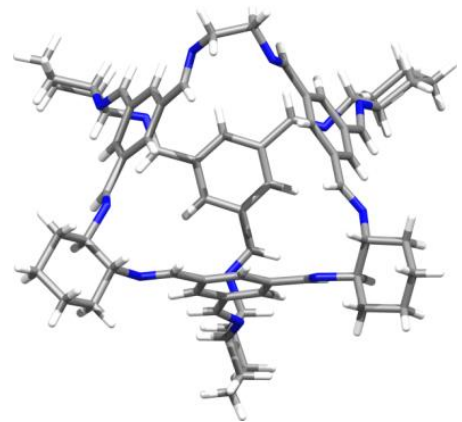

Fig. 4 Single crystal structure for $\mathrm{CC1}^{1} 3^{5}-R \mathrm{MeOH}$ solvate; solvent molecule omitted for clarity. The single ethylenediimine vertex, seen at the top apex of the cage structure, is disordered over four cage vertices in the crystal structure.

This study is the first example of the synthesis of imine cage molecules by dynamic covalent chemistry in a flow reactor. By using the flow reactor to control the reactant ratio, we were able to produce scrambled cage mixtures with a range of physical properties, thus coming a step closer to the goal of obtaining functional materials on demand. The desymmetrised cage, $\mathbf{C C 1}^{1} \mathbf{3}^{5}$, was also isolated, demonstrating that scrambling can be used to access novel POCs, which could be useful substrates for further synthetic modification.

We anticipate that this method will allow the systematic screening for new functional cage molecules, as well as allowing their laboratory scale-up for testing in applications such as adsorption and molecular separations. In the case of soluble cage materials, flow chemistry might be a more convenient method for combinatorial discovery than parallel batch reactors or robotic synthesizers. We have demonstrated the use of two mixed amines, and this could be readily extended to multiple amines, or other reaction components, and hence to the combinatorial optimization or directed evolution of physical properties such as porosity, molecular selectivity, or processability. To give an example, flow reactors might be used to optimize the interfacial and porosity properties of POCs for use as fillers or additives in mixed matrix membranes. ${ }^{31}$

\section{Notes and references}

We thank the Engineering and Physical Sciences Research Council (EPSRC) for financial support under the Grants $\mathrm{EP} / \mathrm{H000925/1}$ (AIC), EP/K009494/1 (SVL) and EP/M004120/1 (SVL), and Pfizer Worldwide Research \& Development (CB). The authors would like to thank EPSRC Dial-a-Molecule Grand 
Challenge Network (EP/K004840/1) for funding a placement with SVL via the Interdisciplinary Mobility Funding scheme (AGS). We also thank Stephen Moss and the MicroBioRefinery for assistance with LC-MS measurements.

1. M. Mastalerz, Angew. Chem. Int. Ed., 2010, 49, 50425053.

2. G. Zhang and M. Mastalerz, Chem. Soc. Rev., 2014, 43, 1934-1947.

3. J. R. Holst, A. Trewin and A. I. Cooper, Nat. Chem., 2010, 2, 915-920.

4. T. Tozawa, J. T. A. Jones, S. I. Swamy, S. Jiang, D. J. Adams, S. Shakespeare, R. Clowes, D. Bradshaw, T. Hasell, S. Y. Chong, C. Tang, S. Thompson, J. Parker, A. Trewin, J. Bacsa, A. M. Z. Slawin, A. Steiner and A. I. Cooper, Nat. Mater., 2009, 8, 973-978.

5. M. J. Bojdys, M. E. Briggs, J. T. A. Jones, D. J. Adams, S. Y. Chong, M. Schmidtmann and A. I. Cooper, J. Am. Chem. Soc., 2011, 133, 16566-16571.

6. M. W. Schneider, I. M. Oppel, H. Ott, L. G. Lechner, H. S. Hauswald, R. Stoll, M. Mastalerz, Chem. Eur. J., 2012, 18, 836-847.

7. L. Chen, P. S. Reiss, S. Y. Chong, D. Holden, K. E. Jelfs, T. Hasell, M. A. Little, A. Kewley, M. E. Briggs, A. Stephenson, K. M. Thomas, J. A. Armstrong, J. Bell, J. Busto, R. Noel, J. Liu, D. M. Strachan, P. K. Thallapally and A. I. Cooper, Nat. Mater., 2014, 13, 954-960.

8. A. Kewley, A. Stephenson, L. J. Chen, M. E. Briggs, T. Hasell and A. I. Cooper, Chem. Mater., 2015, 27, 3207-3210.

9. T. Mitra, K. E. Jelfs, M. Schmidtmann, A. Ahmed, S. Y Chong, D. J. Adams and A. I. Cooper, Nat. Chem., 2013, 5, 276-281.

10. M. Brutschy, M. W. Schneider, M. Mastalerz and S. R. Waldvogel, Adv. Mater., 2012, 24, 6049-6052.

11. M. Brutschy, M. W. Schneider, M. Mastalerz and S. R. Waldvogel, Chem. Commun., 2013, 49, 8398-8400.

12. J. K. Sun, W. W. Zhan, T. Akita and Q. Xu, J. Am. Chem. Soc., 2015, 137, 7063-7066.

13. G. Zhang, O. Presly, F. White, I. M. Oppel and M Mastalerz, Angew. Chem. Int. Ed., 2014, 53, 1516-1520.

14. A. G. Slater and A. I. Cooper, Science, 2015, 348, 988-998.

15. D. J. Cram, Science, 1988, 240, 760-767.

16. J. W. Steed, D. R. Turner and K. J. Wallace, Core concepts in supramolecular chemistry and nanochemistry, John Wiley, Chichester, England; Hoboken, NJ, 2007.

17. N. E. Borisova, M. D. Reshetova and Y. A. Ustynyuk, Chem. Rev., 2007, 107, 46-79.

18. T. Hasell, H. F. Zhang and A. I. Cooper, Adv. Mater., 2012, 24, 5732-5737.

19. M. W. Schneider, I. M. Oppel, A. Griffin and M. Mastalerz, Angew. Chem. Int. Ed., 2013, 52, 3611-3615.

20. G. Jas and A. Kirschning, Chem. Eur. J., 2003, 9, 57085723.

21. R. L. Hartman, J. P. McMullen and K. F. Jensen, Angew. Chem. Int. Ed., 2011, 50, 7502-7519.

22. R. M. Myers, D. E. Fitzpatrick, R. M. Turner and S. V. Ley, Chem. Eur. J., 2014, 20, 12348-12366.

23. S. V. Ley, D. E. Fitzpatrick, R. M. Myers, C. Battilocchio and R. J. Ingham, Angew. Chem. Int. Ed., 2015, 54, 10122 10136.

24. M. E. Belowich and J. F. Stoddart, Chem. Soc. Rev., 2012, 41, 2003-2024.
25. D. P. Lydon, N. L. Campbell, D. J. Adams and A. I. Cooper, Synth. Commun., 2011, 41, 2146-2151.

26. V. Dragone, V. Sans, M. H. Rosnes, P. J. Kitson and L. Cronin, Beilstein J. Org. Chem., 2013, 9, 951-959.

27. M. Kitchin, K. Konstas, M. L. Czyz, O. Valente, C. J. Sumby, M. R. Hill, A. Polyzos and C. J. Doonan, Chem. Commun., 2015, 51, 14231-14234.

$28 . \quad$ S. Jiang, J. T. A. Jones, T. Hasell, C. E. Blythe, D. J. Adams, A. Trewin and A. I. Cooper, Nat. Commun., 2011, 2, 207.

29. S. Klotzbach and F. Beuerle, Angew. Chem. Int. Ed., 2015, 54, 10356-10360.

30. K. Acharyya, S. Mukherjee and P. S. Mukherjee, J. Am. Chem. Soc., 2013, 135, 554-557.

31. A. F. Bushell, P. M. Budd, M. P. Attfield, J. T. A. Jones, T. Hasell, A. I. Cooper, P. Bernardo, F. Bazzarelli, G. Clarizia and J. C. Jansen, Angew. Chem. Int. Ed., 2013, 52, 12531256. 